\title{
Diagnostic Delay in Crohn's Disease: Time for Red Flags
}

\author{
Gionata Fiorino $^{1} \cdot$ Silvio Danese ${ }^{1,2}$
}

Received: 29 August 2016/Accepted: 31 August 2016/Published online: 16 September 2016

(C) Springer Science+Business Media New York 2016

The time between the initial manifestation of a disease and its correct diagnosis is termed diagnostic delay. The significance of diagnostic delay is that the postponement of the institution of effective treatment can profoundly affect patient well-being and overall disease evolution. The diagnosis of Crohn's disease (CD) is often delayed 5-9 months due to the variability and subtlety of its initial manifestations, as opposed to ulcerative colitis (UC), which due to its consistent initial symptoms and manifestations is usually diagnosed more rapidly [1, 2]. Moreover, $\mathrm{CD}$ patients with pure ileal disease and age $<40$ at diagnosis are significantly at risk of considerable diagnostic delay ( $>24$ months) [1], data confirmed by the results of the IMPACT survey among European patients. In this large online survey promoted by the European Federation of Crohn's and Colitis Associations (EFCCA) involving 4990 patients with inflammatory bowel disease (IBD), $20 \%$ of subjects had to wait up to 5 years after symptomatic disease onset in order to receive the correct diagnosis of CD, with consequent impairment of their quality of life [3].

Beyond the immense impact on quality of life, diagnostic delay may also affect the natural history of $\mathrm{CD}$. Several studies indicate that a delayed diagnosis is associated with the development of complications and consequent bowel resection [2, 4-6] Bowel damage increases over time [7], with evolution from non-complicated CD to the development of extensive and irreversible bowel

Silvio Danese

sdanese@hotmail.com

1 Department of Gastroenterology, IBD Center, Humanitas Research Hospital, Rozzano, Milan, Italy

2 Department of Biomedical Sciences, Humanitas University, Via Manzoni 113, 20089 Rozzano, Milan, Italy damage that sometimes occurs quickly. Thia et al. [8] concluded from data gathered from a large populationbased study that the cumulative risk for complications was $18.6 \%$ at 90 days, $22.0 \%$ at 1 year.

In this issue of Digestive Diseases and Sciences Nahon et al. [9] report the results of a prospective multicenter study investigating whether diagnostic delay in CD worsens disease prognosis. The study was conducted in three IBD referral centers in France, enrolling 497 patients diagnosed with CD over a 12-year period (2002-2014). Patients had to recall their symptoms at onset, with the upper quartile of the time interval from symptoms to established diagnosis of the entire cohort defined as diagnostic delay. Poor disease outcome was indicated with at least one of the following events: time to first major intestinal surgery, time to introduction of an immunosuppressant (IMS) and time to first use of an anti-tumor necrosis factor (TNF) agent from disease diagnosis. The development of perianal disease was also assessed.

The median time to diagnosis was 5 months; early diagnosis was defined as $<2$ months, whereas late diagnosis was $\geq 13$ months following the onset of symptoms. Interestingly, delayed diagnosis was associated with a significantly higher rate of major CD-related surgery compared to earlier diagnosis, whereas no differences were reported in the time to first use of IMS or anti-TNF biologic drugs. No predictors (age, sex, disease behavior at diagnosis, or localization) were associated with poor disease outcomes.

This study raises several discussion points including: (1) median time to diagnosis was less than that reported by the Swiss IBD cohort [1], probably related to easier access to the healthcare system in France than in other countries; and (2) the time to diagnosis associated with poor outcomes was $\geq 13$ months, less than the proposed 18-24-month 
therapeutic window of opportunity for early disease $[10,11]$. This study confirms that any delay in CD diagnosis may result in higher surgical risk and probable lower efficacy of therapeutic strategies. Pariente et al. [7] reported that bowel damage as measured by the Lémann index increases according to disease duration. The significantly higher proportion of patients undergoing surgery for bowel damage progression, in the absence of significantly different use of effective therapies in this French cohort supports the hypothesis that diagnostic delay of $\geq 13$ months is associated with a lesser probability of a response to IMS and anti-TNF drugs if given at a late stage. The REACT trial [12] and the SUTD [13] trial demonstrated that early combination of IMS and anti-TNF drugs, although not impacting symptomatic remission rates, decreased the rate of CD-related bowel damage when compared to conventional therapies.

Based on the results of the study by Nahon et al., two key strategies emerge in the management of CD. First, identifying early symptoms and signs of CD by general practitioners and other practitioners not considered IBD specialists is mandatory in order to reduce diagnostic delay so as to diagnose the disease within the therapeutic window of opportunity. With this goal in mind, the ongoing validation of the Red Flags Index [14] may positively impact this feature. Second, the development of new drugs or new therapeutic strategies should focus mainly on the rapid resolution of inflammation and on early prevention of irreversible structural bowel damage, rather than only on symptom control. Facing this challenge from two different sides (early diagnosis and early intervention) may substantially improve disease outcomes over time while positively impacting the overall quality-of-life of CD patients.

\section{References}

1. Vavricka SR, Spigaglia SM, Rogler G, et al. Systematic evaluation of risk factors for diagnostic delay in inflammatory bowel disease. Inflamm Bowel Dis. 2012;18:496-505.
2. Nahon S, Lahmek P, Lesgourgues B, et al. Diagnostic delay in a French cohort of Crohn's disease patients. J Crohns Colitis. 2014;8:964-969.

3. Ghosh S, Mitchell R. Impact of inflammatory bowel disease on quality of life: results of the European Federation of Crohn's and Ulcerative Colitis Associations (EFCCA) patient survey. $J$ Crohns Colitis. 2007;1:10-20.

4. Schoepfer AM, Dehlavi MA, Fournier Net al. Diagnostic delay in Crohn's disease is associated with a complicated disease course and increased operation rate. Am J Gastroenterol. 2013; 108:1744-1753; quiz 1754.

5. Pellino G, Sciaudone G, Selvaggi F, Riegler G. Delayed diagnosis is influenced by the clinical pattern of Crohn's disease and affects treatment outcomes and quality of life in the long term: a cross-sectional study of 361 patients in Southern Italy. Eur J Gastroenterol Hepatol. 2015;27:175-181.

6. Li Y, Ren J, Wang G, et al. Diagnostic delay in Crohn's disease is associated with increased rate of abdominal surgery: a retrospective study in Chinese patients. Dig Liver Dis. 2015;47: 544-548.

7. Pariente B, Mary JY, Danese S, et al. Development of the Lemann index to assess digestive tract damage in patients with Crohn's disease. Gastroenterology. 2015;148:e53.

8. Thia KT, Sandborn WJ, Harmsen WS, Zinsmeister AR, Loftus EV Jr. Risk factors associated with progression to intestinal complications of Crohn's disease in a population-based cohort. Gastroenterology. 2010;139:1147-1155.

9. Nahon, S, Lahmek, P, Paupard, T, Lesgourgues, B, Chaussade, S, Peyrin-Biroulet, L, Abitbol, V. Diagnostic delay is associated with a greater risk of early surgery in a french cohort of Crohn's disease patients. Dig Dis Sci. (Epub ahead of print). doi:10.1007/ s10620-016-4189-z.

10. Peyrin-Biroulet L, Loftus EV Jr, Colombel JF, Sandborn WJ. Early Crohn disease: a proposed definition for use in diseasemodification trials. Gut. 2010;59:141-147.

11. Danese S, Fiorino G, Fernandes C, Peyrin-Biroulet L. Catching the therapeutic window of opportunity in early Crohn's disease. Curr Drug Targets. 2014;15:1056-1063.

12. Khanna R, Bressler B, Levesque BG, et al. Early combined immunosuppression for the management of Crohn's disease (REACT): a cluster randomised controlled trial. Lancet. 2015;386:1825-1834.

13. D'Haens G, Baert F, van Assche G, et al. Early combined immunosuppression or conventional management in patients with newly diagnosed Crohn's disease: an open randomised trial. Lancet. 2008;371:660-667.

14. Danese S, Fiorino G, Mary JY, et al. Development of red flags index for early referral of adults with symptoms and signs suggestive of Crohn's disease: an IOIBD initiative. J Crohns Colitis. 2015;9:601-606. 